\title{
On-line laser radiation controlled to the removal of adhesive on teeth after bracket debonding
}

\author{
Clara Gómez ${ }^{1}$,Juan Carlos Palma ${ }^{2}$, Ángel Costela ${ }^{1}$ \\ 1: Departamento de Sistemas de Baja Dimensionalidad, Superficies y Materia Condensada, \\ Instituto de Química Física Rocasolano, CSIC, Madrid, Spain. \\ 2: Departamento de Estomatología IV, Facultad de Odontología, UCM, Madrid, Spain.
}

\begin{abstract}
Background and aims: After bracket debonding a correct removal of the adhesive from tooth surfaces without causing any iatrogenic damage to the enamel is needed. However, conventional techniques do not allow a selective removal process. The present article focuses on the removal of adhesive on teeth after bracket debonding by using laser radiation at $355 \mathrm{~nm}$ (third harmonic wavelength of a Q-switched Nd:YAG).

Material and methods: Brackets were bonded to 10 extracted human premolars from young patients and removed after a storage period of 2 months. As real-time diagnostic technique, laserinduced breakdown spectroscopy (LIBS) elemental analysis was applied for precisely controlling the removal of the adhesive and morphological analysis of the etched surfaces was carried out by scanning electron microscopy (SEM).

Results: LIBS technique allowed an on-line precise control in the adhesive removal process. SEM analysis revealed the capability of the $355 \mathrm{~nm}$ UV laser radiation to complete the removal of the adhesive on the tooth with no signs of damage on the enamel.
\end{abstract}

Conclusions: Laser ablation process at $355 \mathrm{~nm}$ monitored by the LIBS technique allows to carry out efficient removal of the adhesive on teeth.

Key words: laser ablation $\cdot$ adhesive $\cdot$ laser-induced breakdown spectroscopy • bracket debonding $\cdot$ orthodontics

\section{Introduction}

After removal of orthodontic attachments, the primary orthodontic goal is to return the enamel surface to its original state ${ }^{1)}$. The main objective of bracket debonding is the removal of the adhesive resin from tooth surfaces without iatrogenic damage to the enamel structure ${ }^{2,3}$. Cleaning with abrasives before etching, acid etching, forcibly removing brackets or mechanical removal of remaining composite by the use of rotary instruments are responsible of possible damages or

Addressee for Correspondence:

Clara Gómez (*Corresponding author)

Departamento de Sistemas de Baja Dimensionalidad, Superficies y Materia Condensada

Instituto de Química Física Rocasolano

Consejo Superior de Investigaciones Científicas, CSIC

C/Serrano 119, 28006 Madrid, Spain

Phone number: +34917459532 Fax: + 915642431

c.gomez@iqfr.csic.es even fractures to enamel ${ }^{4,5)}$. In order to eliminate potential plaque retention and to restore the aesthetic appearance of the enamel surface, a correct adhesive removal is necessary ${ }^{6}$ ).

Researchers have tested different techniques for resin removal and enamel polishing. The most popular tools to remove the adhesive resin are tungsten carbide burs which seem to be faster and more effective than Sof-Lex discs, ultrasonic tools, hand instruments, rubbers or composite burs ${ }^{7,8)}$. Nevertheless, tungsten carbide bur requires multistep polishing 7 ).

Over the last years, the possibility of using laser ablation as an alternative method to the conventional techniques of mechanical or chemical removal has been taken into account 9,10 ). It is important to point out that a selective removal of the material without damaging the substrate and accurately controlling the

Received date: November 15th, 2016 Accepted date: December 21st, 2016 
depth of the removed layer is possible due to the different and characteristic absorption at the laser wavelength exhibited by each substrate ${ }^{11)}$. Combination of the laser ablation process with the use of analytical techniques, allows to identify the substrate, carry out a layer analysis of the surface and control the removal process in real time ${ }^{12}$ ). One such technique is laserinduced breakdown spectroscopy (LIBS), which can be utilized for spectroscopic analysis of the emission from the plasma generated when the laser radiation interacts with the surface of the material 13, 14). Laser ablation complemented with the LIBS technique could be an efficient clinical method of returning enamel to as near its original condition as possible after brackets removal. The wavelength, duration and energy of the laser pulse as well as the properties of the material clearly influence the mechanism responsible for the laser removal process. When the ablation process uses UV wavelength, it seems to be based in a photoablative mechanism where absorption of one or more photons results in an electronic excitation followed by decomposition of the compound caused by direct bond breaking in the solid, in competition with various relaxation processes ${ }^{15)}$. An on-line control of the process is more easily implemented when the ablation process is based on this mechanism than when photothermal mechanisms are predominant.

In the literature there are some works investigating the use of several types of laser radiation to remove adhesion resin after bracket debonding ${ }^{16}$ ). Most of these studies use lasers operating in the infrared (IR) region of the electromagnetic spectrum, and both positive ${ }^{17-19)}$ and negative results are reported 20,21 ).

Nowadays efforts are made to find tools and methods for complete removal of adhesive remnants, minimizing enamel loss and achieving a smooth surface after the completion of orthodontic treatment with fixed appliances. With this aim, we tested the use of UV laser radiation, which allows a more precise and thus more easily controlled ablation process to remove adhesive remnant after brackets debonding without damaging the tooth surface. In addition, we implemented a flexible and user-friendly detection system that comprises a non-intensified CCD camera to properly control the removal process of the adhesive.

\section{Materials and methods}

\section{Samples preparation}

10 human premolars from young patients (between 12 and 15 years old), extracted for different orthodontic issues, were thoroughly debrided and stored in an aqueous solution of thymol ( 0.1 per cent) at room temperature to prevent dehydration. Teeth were rinsed before the start the experiment and then bonded according to the manufacturers' instructions: the buccal enamel was etched for $15 \mathrm{~s}$ with a $35 \%$ phosphoric acid (Scotchbond ${ }^{\mathrm{TM}}$ Etchant Phosphoric Acid from 3M ESPE, MN, USA), rinsed with air-and-water spray, and air dried for $20 \mathrm{~s}$, and sealed with Transbond ${ }^{\text {TM }}$ Liquid (3M Unitek, CA, USA). Transbond ${ }^{\mathrm{TM}}$ XT adhesive (3M Unitek) was applied onto the bracket pad, and the bracket was positioned on the prepared enamel; a microbrush was used to remove any excess. The adhesive was light-cured with a $3 \mathrm{M}$ Unitek Ortholux XT Visible Light Curing Unit for resin polymerization for 5 $s$ at each side (left and right). The brackets used in this study had 0.018 inch slots (Forestadent, Pforzheim, Germany). Finally, the teeth were stored in a humid chamber at a temperature of $37^{\circ} \mathrm{C}$ for two months.

\section{Bracket removal}

The brackets were debonded by gently squeezing with pliers (Weingart Utility Pliers, 3M Unitek).

\section{Adhesive resin removal}

The removal of adhesive resin with UV radiation was performed by using a Q-switched Nd:YAG laser (Spectron SL803G), which produces pulses at $355 \mathrm{~nm}$, with $6.2 \mathrm{~mJ}$ energy, $\sim 5 \mathrm{~ns}$ pulse duration (FWHM) and pulse repetition rate was set at $1.25 \mathrm{~Hz}$ (see Table 1).

Table 1: Laser system description and summary of the laser parameters used in this study.

Laser system

Commercial name: Q-switched Nd:YAG (Spectron SL 803G)

Manufacturer: Spectron Laser Systems

Laser classification according to laser medium: Solid-state laser

Laser classification according to safety: 4

Laser Parameters

Wavelength: $355 \mathrm{~nm}$

Irradiation mode: pulsed wave (pw)

Pulse duration: $\sim 5 \mathrm{~ns}$

Energy output: $6.2 \mathrm{~mJ}$

Pulse power: $1.24 \cdot 10^{6} \mathrm{~W}$

Fluence: $0.8 \mathrm{~J} / \mathrm{cm}^{2}$

Diameter of the spot: $1 \mathrm{~mm}$

Repetition rate used: $1.25 \mathrm{~Hz}$ 
The laser beam passed through a spherical lens of focal length $\mathrm{f}=5 \mathrm{~cm}$ placed at $6.5 \mathrm{~cm}$ of the sample. The focused laser beam was directed onto the teeth which were placed normal to the beam onto a X-Y translation stage. A pyroelectric detector Gentec-DE $500+$ (in combination with Gentec-EO SOLO console) allowed to perform energy measurements of the laser pulse incident on the adhesive on the teeth. On the laser path, a quartz beam spliter directed $\sim 10 \%$ of the beam energy to a photodiode allowing the incident energy in the adhesive on the teeth being continuously monitored during the experiments (Figure 1).

LIBS was employed in the elemental analysis of both ablated adhesive and enamel. Light from the ablation plume was collected by an optical fiber bundle (19 fibers of $200 \mu \mathrm{m}$ each) coupled to the entrance slit of a high resolution spectrograph $(0.300$ Meter Focal Length Triple Grating Imaging Monochromator/Spectrograph, ARC SpectraPro300i). A grating of 300 grooves $/ \mathrm{mm}$ with a wavelength coverage of $300 \mathrm{~nm}$ was employed. The spectrograph was coupled to a non-intesified CCD detector (Spectru MM:GS128B, Acton Research, 1024 x 122 imaging array, $24 \mu \mathrm{mx} 24 \mu \mathrm{m}$ pixels). The signal generated by a photodiode (EGC-100) sampled with a boxcar (Stanford Research Model 250) controlled the timing of the data acquisition system. Figure 1 shows a schematic of the experimental LIBS set-up used.

\section{Morphological analysis}

In order to complete the characterization of the abla-

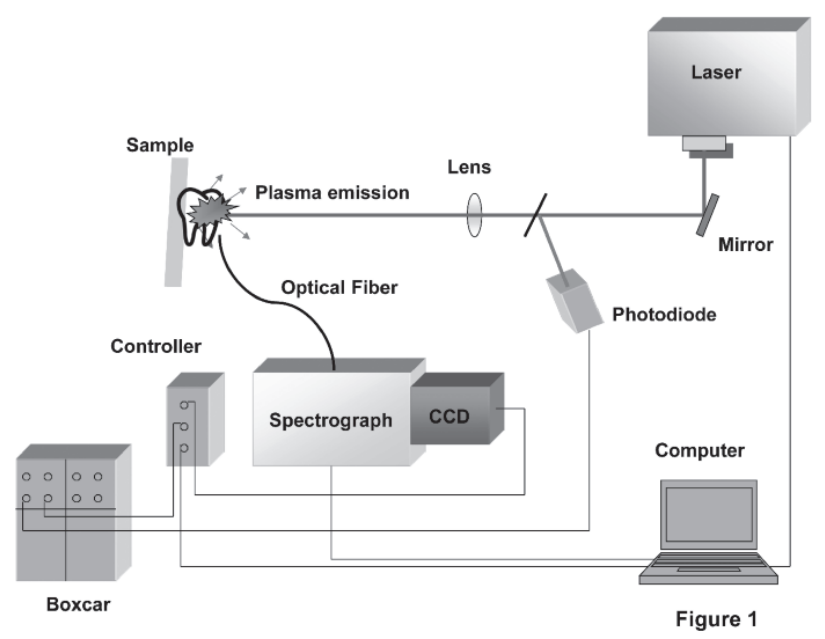

Figure 1: Scheme of the experimental set-up used for the LIBS experiments. tion process of the adhesive, a morphological analysis of the irradiated zones was performed using a scanning electron microscopy (SEM) with a (FE-SEM) SU 8000 , Hitachi microscope after metallization of the samples with gold.

\section{Results}

Characteristic emission lines both of the enamel and adhesive under irradiation at $355 \mathrm{~nm}$ as well as their elemental assignation ${ }^{22}$ ) are collected in Table 2.

Figure 2 shows LIBS spectra of enamel and adhesive. Characteristic lines of $\mathrm{Ca}$ are dominant in the emission spectrum of the enamel. In the case of the adhesive, the dominant characteristic peaks correspond to Si and Ti.

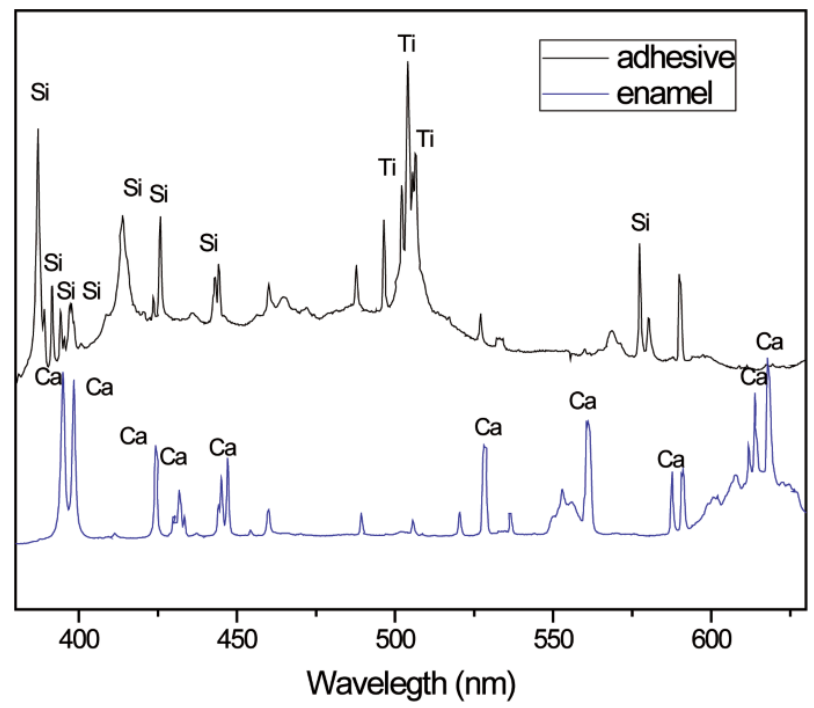

Figure 2: LIBS spectra of enamel and adhesive. Irradiation wavelength $355 \mathrm{~nm}$.

Table 2: Main atomic lines (nm) identified in the LIBS spectrum of the adhesive and enamel at the irradiation wavelength at $355 \mathrm{~nm}$

\begin{tabular}{cl}
\hline Adhesive & \multicolumn{1}{c}{ Enamel } \\
\hline & \\
$386.26 ; 390.55 ; 395.57 ;$ & $394.89 ; 397.37 ; 422.67 ;$ \\
$399.18 ; 414.92 ; 421.24 ;$ & $443.50 ; 445.59 ; 527.03 ;$ \\
$441.17 ; 577.21(\mathrm{Si})$ and & $551.30 ; 558.20 ; 559.85 ;$ \\
$501.42 ; 503.99 ; 506.46(\mathrm{Ti})$ & $585.57 ; 612.22 ; 616.96(\mathrm{Ca})$
\end{tabular}


Figure 3 shows an example of adhesive removal in tooth with the number of pulses monitored by the LIBS technique. Emission lines from the adhesive progressively disappear with the number of pulses whereas emissions lines of $\mathrm{Ca}$ characteristic from enamel begin to appear.

In order to characterize the ablation process, a morphological analysis by SEM of the irradiation zones was carried out. Figures $\mathbf{4 a}, \mathbf{4} \mathbf{b}, \mathbf{4} \mathbf{c}$ show the complete removal of adhesive on tooth after three pulses of laser radiation at $355 \mathrm{~nm} ; 0.8 \mathrm{~J} / \mathrm{cm}^{2}$. Figure $4 \mathbf{c}$ also serves as control image of the enamel before bonding for comparing the effect of the laser on the enamel morphology. This figure shows a strip of adhesive after removal of the bracket with pliers, with some laser pulses. At the top of the strip, enamel that has supposedly remained intact is observed, where pliers, at the same time, have both removed bracket and adhesive. At the low of the strip, enamel that was outside the area that occupied the bracket can be observed. It is thus corroborated that with an adequate number of laser pulses we completely eliminate the adhesive without damaging the enamel.

\section{Discussion}

Elemental analysis of the ablated materials from both adhesive and enamel was carried out by means of the LIBS technique, which allows on-line control of the ablation process. Enamel with no adhesive on it was

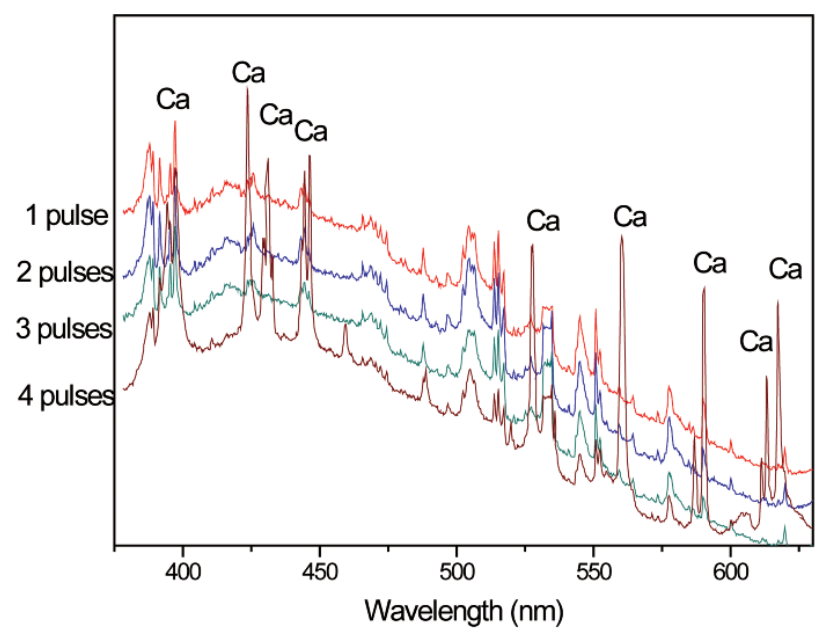

Figure 3: Evolution of LIBS spectrum with the number of pulses during the removal of the adhesive from the tooth. Irradiation wavelength: $355 \mathrm{~nm}$. first irradiated to characterize its plasma emission. Characteristic lines of $\mathrm{Ca}$ are dominant in the emission spectrum of the enamel. Next, LIBS analysis was repeated with just adhesive on the enamel. However, in the emission spectrum of the adhesive dominant
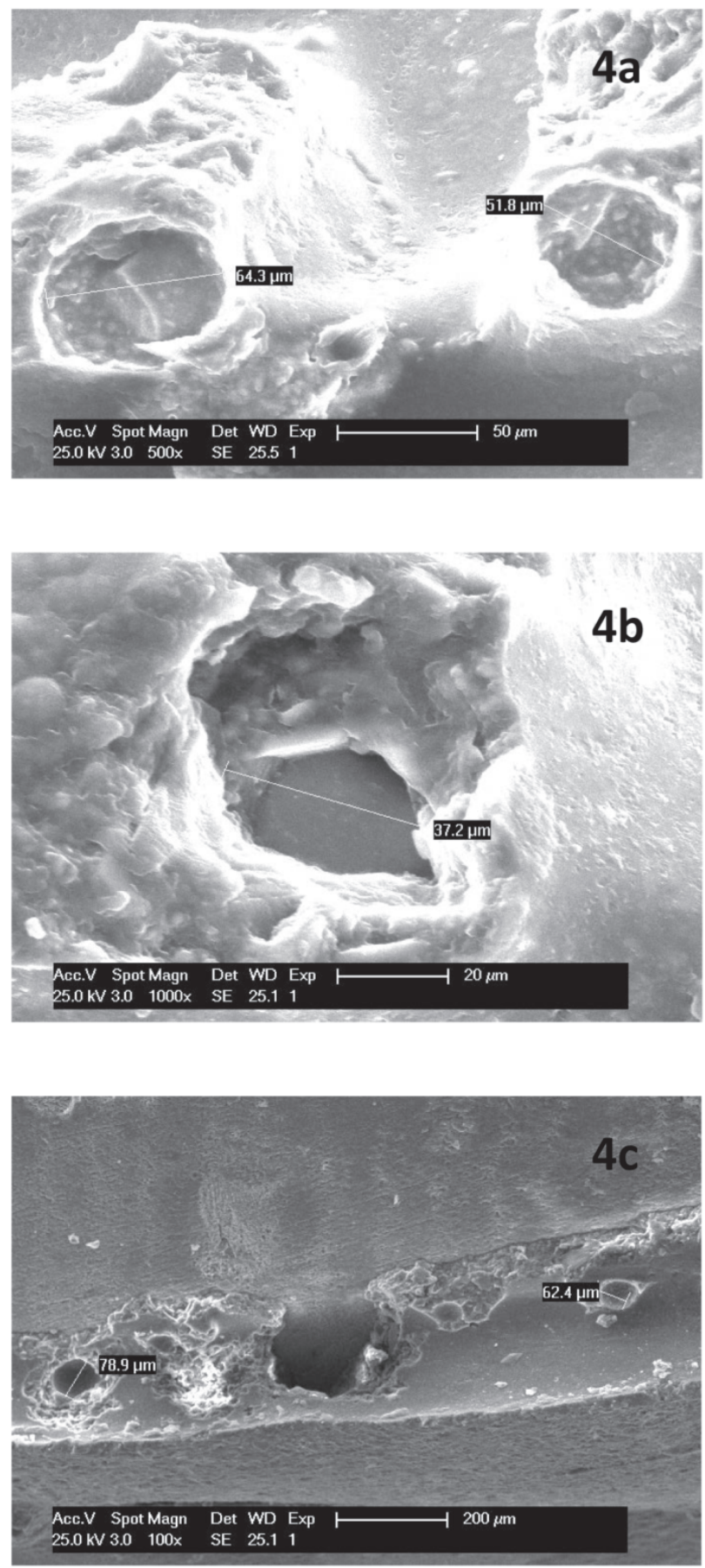

Figure 4: Scanning electron micrographs showing the removal of adhesive on tooth after three pulses of laser radiation at $355 \mathrm{~nm} ; 0.8 \mathrm{~J} / \mathrm{cm}^{2}$. 
characteristic peaks correspond to $\mathrm{Si}$ and Ti. Thus, LIBS spectra obtained from both adhesive an enamel, are clearly different confirming the appropriateness and potential of this technique for identifying and distinguish between different substrates.

The LIBS technique allows an on-line automated control of the ablation process to remove adhesive from teeth by analyzing the plasma generated in each incident pulse. In this way, when characteristic peaks of the adhesive totally disappear and an emission peak from the enamel is detected, the ablation process must stop in order to avoid any damage to the surface, as can be seen in the Figure 3 .

In addition, the morphological analysis by SEM of the irradiation zones confirms that the ablation of the adhesive at the wavelength used $(355 \mathrm{~nm})$ causes neither material deposition nor chemical, thermal or mechanical change of the region adjacent to the irradiated area. The adhesive was completely removed after 3 pulses at fluency of $0.8 \mathrm{~J} / \mathrm{cm}^{2}$ and the enamel was not damaged. An adequate clean-up of the adhesive without enamel loss is difficult to achieve with conventional methods (scraping with a scaler or band-removing plier or a tungsten carbide bur in a contra-angle hand piece and the use of abrasive discs) ${ }^{5)}$. The outer layer of enamel contains more minerals and fluoride than the deeper layers, so, there is an increased risk of decalcification as the consequence of the damage to the enamel surface by the use of these devices to remove the adhesive. In addition, enamel roughness (increased by the method used to remove the adhesive) is related to the accumulation and retention of bacterial plaque ${ }^{23}$ ). In our study, the laser radiation employed here complemented with the LIBS technique allows a selective removal process that prevents decalcification and avoids an increase in the roughness of enamel, thus making unnecessary subsequent polish-

\section{References}

1: Campbell PM (1995): Enamel surfaces after orthodontic bracket debonding. The Angle Orthodontist, 65:103-110

2: Joo HJ, Lee YK, Lee DY, Kim YJ, Lim YK (2011): Influence of orthodontic adhesives and clean-up procedures on the stain susceptibility of enamel after debonding. The Angle Orthodontist, 81: 334-340.

3: Suliman SN, Trojanb TM, Tantbirojnc D, Versluisd A (2015): Enamel loss following ceramic bracket ing methods and ultimately eliminating the possibility of accumulation of plaque.

It should be pointed out that the gaussian pulse shape of the laser here employed is reflected in the images in Figure 4, where it is seen that the average area removed has initially $58.92 \mu \mathrm{m}$ of diameter, which is decreasing as we move away from the surface that was in contact to the bracket. A top hat beam with a uniform fluency within the spot would be more suitable for this application. Scanning the beam along the surface with the adhesive so that every three shots the beam is moved to an adjacent position will allow complete removal of the adhesive without any damage to the enamel.

\section{Conclusions}

Laser ablation process at $355 \mathrm{~nm}$ allows to carry out efficiently removal of adhesive on teeth after bracket debonding. The suitability of the LIBS technique to continuously monitoring the removal process is demonstrated, which allows an on-line precise control of the adhesive removed. As major advantages of this procedure can be highlighted the good signal-to-noise ratio and that no especial requirements are necessary to the alignment of the detection system, which allows using LIBS technique as a real-time user-friendly diagnostic technique for the laser ablation process. Morphological studies showed the capability of the 355 nm UV laser radiation to complete removal of the adhesive on the tooth without damage to the enamel structure.

\section{Author Disclosure Statement}

No competing financial interest exist. debonding: a quantitative analysis in vitro. The Angle Orthodontist, 85:651-656.

4: Hosein I, Sherriff M, Ireland AJ (2004): Enamel loss during bonding, debonding, and clean up with use of a self-etching primer. American Journal of Orthodontics and Dentofacial Orthopedics 126:717724.

5: Eliades T, Gioka C, Eliades G, Makou M (2004): Enamel surface roughness following debonding using two resin grinding methods. European 
Journal of Orthodontics, 26:333-338.

6: Ryf S, Flury S, Palaniappan S, Lussi A, van Meerbeek B, Zimmerli B (2012): Enamel loss and adhesive remnants following bracket removal and various clean-up procedures in vitro. European Journal of Orthodontics, 34:25-32.

7: Janiszewska-Olszowska J, Szatkiewicz T, Tomkowski R, Tandecka K, Grocholewicz K (2014): Effect of orthodontic debonding and adhesive removal on the enamel - current knowledge and future perspectives - a systematic review. Medical Science Monitor, 20: 1991-2001.

8: Karan S, Kircelli BH, Tasdelen B (2010): Enamel surface roughness after debonding. The Angle Orthodontist, 80:1081-1088.

9: Lu YF, Takai M, Komuro S, Shiokawa T, Aoyagi Y (1994): Surface cleaning of metals by pulsed-laser irradiation in air. Applied Physics A, 59: 281 288.

10: Tsunemi A, Hagiwara K, Saito N, Nagasaka K, Miyamoto Y, Soto O, Tashiro H (1996): Complete removal of pain from metal surface by ablation with a TEA CO2 laser. Applied Physics A, 63: 435439.

11: Gómez C, Costela A, García-Moreno I, Sastre R (2006): Comparative study between IR and UV laser radiation applied to the removal of graffitis on urban buildings. Applied Surface Science, 252: 2782-2793.

12: Castillejo M, Martín M, Oujja M, Silva D, Torres R, Manousaki A, Zafiropulos V, Van den Brink OF, Heeren RMA, Teule R, Silva A, Gouveia H (2002): Analytical study of the chemical and physical changes induced by $\mathrm{KrF}$ laser cleaning of tempera paints. Analytical Chemistry, 74: 4662-4671.

13: Knight AK., Scherbarth NL, Cremers DA, Ferris MJ (2000): Characterization of Laser-Induced Breakdown Spectroscopy (LIBS) for Application to Space Exploration. Applied Spectroscopy, 54: 331340.

14: Yueh F, Singh JP, Zhang H: Laser induced Breakdown Spectroscopy, elemental analysis. In:
(Meyers RA,ed) Encyclopedia of Analytical Chemistry. 2000, John Wiley \& Sons, New York, pp 2066-2087.

15: Niemz MH. Laser-Tissue Interactions; Fundamentals and Applications. 2004, $3^{\text {rd }}$ ed. New York: Springer-Verlag, Berlin, Heidelberg, pp. 45-150.

16: Thomas BW, Hook CR, Draughn RA (1996): Laseraided degradation of composite resin. The Angle Orthodontics, 66:281-286.

17: Yassaei S, Soleimanian A, Nik ZE (2015): Effects of Diode Laser Debonding of Ceramic Brackets on Enamel Surface and Pulpal Temperature. The Journal of Contemporary Dental Practice, 16:270274.

18: Smith SC, Walsh LJ, Taverne AA (1999): Removal of orthodontic bonding resin residues by $\mathrm{CO} 2$ laser radiation: surface effects. Journal of Clinical Laser Medicine and Surgery, 17:13-18.

19: Dostalova T, Jelinkova H, Remes M, Ś̉ulc J, Nĕmec M (2016): The use of the Er:YAG laser for bracket debonding and its effect on enamel damage. Photomedicine and Laser Surgery, 9: 294-399.

20: Yassaei S, Aghili H, Joshan N (2015): Effects of removing adhesive from tooth surfaces by Er:YAG laser and a composite bur on enamel surface roughnessand pulp chamber temperature. Dental Research Journal (Isfahan), 12: 254-259.

21: Ahrari F, Akbari M, Akbari J, Dabiri G (2013): Enamel surface roughness after debonding of orthodontic brackets and various clean-up techniques. Journal of Dentistry of Tebran University of Medical Science, 10: 82-93.

22: D.R. Lide (Ed.). CRC Handbook of Chemistry and Physics, 76 ${ }^{\text {th }}$ ed., CRC Press, Boca Raton, FL (19951996).

23: Bollen CM, Lambrechts P, Quirynen M (1997) Comparison of surface roughness of oral hard materials to the threshold surface roughness for bacterial plaque retention: a review of the literature. Dental Materials, 13:258-269.

\section{[Acknowledgements]}

This research was supported by the Spanish Research Project MICINN (Ref.:MAT2014-51937-C3-1-P). The authors report that they have no conflicts of interest related to the present study. 